\title{
Development of Postural Control During the First 18 Months of Life
}

\author{
Mijna Hadders-Algra \\ Department of Neurology, Developmental Neurology, University Medical Center \\ Groningen, University of Groningen, Groningen, the Netherlands
}

\begin{abstract}
SUMMARY
The present paper reviews the development of postural adjustments during infancy. In the control of posture, two functional levels can be distinguished. The basic level deals with the generation of direction-specific adjustments, meaning that dorsal muscles are primarily activated when the body sways forward, whereas ventral muscles are primarily activated when the body sways backward. The second level is involved in adaptation of the direction-specific adjustments. Postural development starts with a repertoire of direction-specific adjustments suggesting that the basic level of control has an innate origin. At first, during the phase of primary variability, postural activity is largely variable and can be minimally adapted to environmental constraints. At 3 months, postural activity shows a transient period during which few postural muscles participate in postural activity. From 6 months onward, the phase of secondary variability starts, during which the second level of postural control becomes functionally active and infants develop the ability to adapt postural activity to the specifics of the situation. Initially, adaptation can be accomplished in a simple way only, but from 9-10 months onward, it can be performed by the subtle adaptation of the degree of muscle contraction. Around 13-14 months, anticipatory
\end{abstract}

Reprint requests to: Mijna Hadders-Algra, Developmental Neurology, University of Groningen, Groningen, The Netherlands; e-mail: m.hadders-algra@med.rug.nl postural adjustments emerge. It is concluded that the development of postural adjustments is characterized by four periods of transition occurring at the ages of $3,6,9-10$, and 13-14 months. The major transition occurs at 6 months, when infants move from the phase of non-adaptive, primary variability to the phase of ada ptive, secondary variability.

\section{KEYWORDS}

motor development, infant, variability, transition, anticipatory postural control

\section{INTRODUCTION}

Postural control primarily serves two goals. First, postural control aims at the maintenance of balance, which means that under static conditions, the center of pressure and the projection of the center of gravity remain inside the support surface. The other goal is to form an interface between perception and action (Massion et al., 2004). The control of posture in the human is characterized by complexity, which has its roots in phylogeny (Gramsbergen, 2005). In the course of phylogeny, the upper limbs became increasingly involved in skilled manual tasks and decreasingly in postural control. Quadrupedal stance and gait were exchanged for bipedal stance and gait. This shift had important implications for postural control because it was associated with a substantial reduction in the base of support. Virtually all parts of the 
nervous system became involved in the control of posture (Dietz, 1992; Massion, 1992; Massion et al., 1999). The complex and distributed organization of human postural control explains why the development of postural control in the human takes many years, i.e., at least until adolescence (Schmitz et al., 2002; Van der Heide et al., 2003; Roncesvalles et al., 2004). The complex nature of the control system induces vulnerability for dysfunctions in case of adverse conditions during early life, such as a pre- or perinatally acquired lesions of the brain or preterm birth (see the contributions of Brogren et al., Fallang \& HaddersAlgra; Geuze; Van der Heide \& Hadders-Algra, and Woollacott, this issue).

In the task of controlling posture, the nervous system is faced with the problem of dealing with a redundancy in degrees of freedom, due to the multitude of participating muscles and joints. Bernstein (1935) suggested that the motor problem posed by the surplus in degrees of freedom might be solved by organizing motor output with the help of synergies. Synergies enable the nervous system to reduce the number of afferent signals needed to generate and guide an ongoing movement and to reduce the number of efferent activities involved in motor control. This means that in movement control, the brain does not specify each single muscle contraction but that it uses a repertoire of neuronal representations of movements with prestructured motor commands. The nervous system indeed organizes postural control with the help of synergies-flexible synergies, which can be finetuned to task-specific conditions (e.g., Horak \& Nashner, 1986; Hirschfeld \& Forssberg, 1992; Macpherson, 1994, Massion et al., 2004).

Forssberg and Hirschfeld (1994), who studied postural adjustments in sitting adults, formulated a functional model on the organization of postural adjustments-the so-called central pattern generator (CPG) model. In general, CPG-activity is used to describe the neural organization of rhythmical movements like locomotion, respiration, and mast- ication. The CPGs refer to neural networks coordinating the activity of many muscles. The activity level in these networks is controlled by reticulospinal neurons, whereas segmental afferent input results in a modulation of the output pattern (Grillner et al., 1995). Essential to the CPG-model for postural adjustments is its organization in two levels. The first level is involved in the generation of the basic direction-specific response pattern. Direction-specificity means that perturbations inducing a forward sway of the body elicit a response pattern in the muscles on the dorsal side of the body, whereas perturbations inducing a backward body sway evoke responses in the 'ventral' muscles.

The second level is involved in the fine-tuning of the basic response pattern, based on multisensorial afferent input from somatosensory, visual, and vestibular systems. Such modulation can be achieved in various ways, for instance, by changing the order in which the agonist muscles are recruited (e.g., in a caudo-to-cranial sequence or in a reverse order), by altering the degree of antagonist activation, or by modifying the size of the muscle contraction (EMG-amplitude).

The aim of the present paper is to review the available knowledge on the development of postural adjustments during infancy. One of the central themes in the review is variability. Gradually it has become clear that typical motor development is characterized by two phases of variability (Neuronal Group Selection Theory-Edelman 1989, Touwen 1993, Hadders-Algra 2000). Development starts with the phase of primary variability, during which variation in motor behavior is not geared to external conditions. Next, the phase of secondary variability takes over, during which motor performance can be adapted to specific situations. The present review indicates that in the development of postural adjustments, four moments of transition can be distinguished, occurring around the ages of 3, 6, 910 , and 13-14 months of age. The transition at 6 months probably is the major one, in which postural 
control moves from the phase of primary variability to the phase of secondary or adaptive variability.

\section{FROM BIRTH TO SIX MONTHS: PRIMARY VARIABILITY IN DIRECTION-SPECIFIC ADJUSTMENTS}

\section{An innate repertoire of direction-specific postural adjustments}

Hedberg et al. (2004) were the first to study systematically postural adjustments in very young infants. The results of their study indicate that at the age of 1 month, infants can generate directionspecific postural adjustments, meaning that the basic level of postural control is functionally active at this age and possibly has an innate origin. Hedberg et al. assessed postural adjustments by means of external perturbations in a sitting position. In subjects of 5 months or older, the trigger to producing direction-specific muscle activity in this condition is the somatosensory information generated by pelvis rotation (Forssberg \& Hirschfeld, 1994; Hadders-Algra et al., 1996a). The data of Hedberg et al. indicated, however, that in 1-month-old infants, the sensory information generated by pelvis rotation is insufficient to trigger direction-specific postural activity. Additionally, vestibular information cannot serve as a primary trigger of postural activity because the data indicate that prior to perturbation the head variably sways in all directions. Therefore, the authors hypothesized that possibly multiple sources of sensory information from the pelvic region-such as proprioceptive information and tactile information generated by stretch-sensitive mechanoreceptors in the buttock region-cooperate in triggering postural activity.

At early age, postural activity is characterized by a large variation in direction-specific postural patterns. The variation is especially apparent in the combinations in which postural muscles are activated, i.e., infants may activate one, two, or more direction-specific muscles in any combination. In infants older than 1 month, the number of directionspecific muscles that participate in the directionspecific adjustments first decreases with increasing age, reaching its nadir at the age of 3 months (Hedberg et al., [accepted for publication] 2005). The reduced expression of direction-specificity around the age of 3 months might explain why previous researchers had difficulty in finding direction-specific postural adjustments at this age (Woollacott et al., 1987; Harbourne et al., 1993). The finding that postural muscle activity is low around 3 months fits the findings of EMGrecordings of spontaneous motor behavior (HaddersAlgra et al., 1992) and H-reflex studies (Hakamada et al., 1988), indicating that motoneuron excitability decreases during the first 3 months after birth.

After the age of 3 months, the number of direction-specific muscles recruited in the postural adjustments increases again. The data of Hedberg et al. (2005) showed that after the transitional period of low postural activity at 3 months, postural muscle activation rates during sitting were significantly more related to achievements in spontaneous motor behavior than prior to the transitional period. These findings suggest that the age of 3 months is a period of developmental transition in postural control. In fact, others had already indicated that 3 months can be considered an age of major neuro-developmental transition (e.g., Prechtl, 1984). Three months is the age at which functional activity in the basal ganglia, cerebellum and parietal, temporal, and occipital cortices increases substantially (Rubinstein et al., 1989; Chugani, 1998). Three months is also the age at which the quality of general movements has considerable predictive power for later developmental disorders (Hadders-Algra, 2004). In addition, the period of transition is the age at which goaldirected arm motility emerges. 


\section{Transition from primary to secondary variability}

The first goal-directed reaching movements do not end in successful grasping, but from the age of 4 to 5 months, they do (Touwen, 1976). As soon as reaching results in successful grasping, it is accompanied by direction-specific postural adjustments (Van der Fits et al., 1999a). From 3 to 6 months, infants continue to show a variable repertoire of direction-specific adjustments. Characteristic for this period is that postural activity can be adapted to a minimal extent only to the specific situation-for instance to the position of the infant (supine versus sitting; Van der Fits et al., 1999a). The capacity to adapt postural activity significantly emerges at the age of 6 months, as illustrated by two findings.
First, it has been shown that from 6 months onward, infants develop the capacity to select from the repertoire of direction-specific adjustments the pattern in which all direction-specific muscles are activated ('en bloc pattern'; Hadders-Algra et al., 1996a; Van der Fits et al., 1999b; Fig. 1). A randomized training study indicated that selection occurs from experience (Hadders-Algra et al., 1996b). Infants explore, by means of active trial and error, which direction-specific postural pattern results in the best stabilization of the head in space (Hadders-Algra et al., 1996a). During infancy, this pattern is called the en bloc pattern.

Second, it has been demonstrated that from 6 months onward, infants are able to adapt the selection of the en bloc pattern to the degree of balance

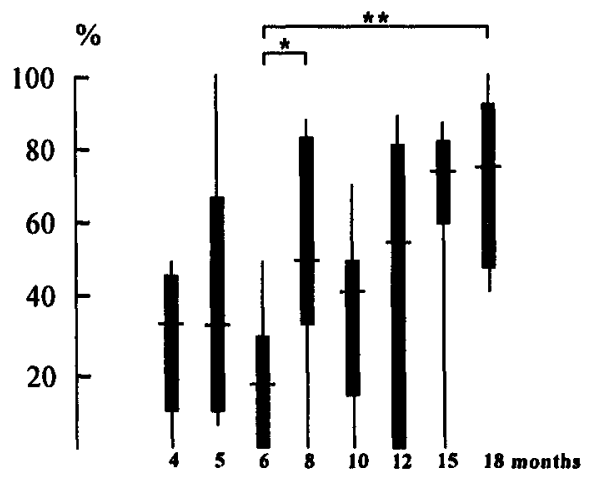

Fig. 1: Rates of the occurrence of the direction specific en bloc 'NE + LE' pattern during reaching in supported sitting condition in ten typically developing infants studied longitudinally. $\mathrm{NE}=$ neck extensor muscle, $\mathrm{LE}=$ lumbar extensor muscle. Data are presented by ranges (vertical bars), interquartile ranges (boxes), and median values (horizontal bars). ${ }^{*}=p<0.05,{ }^{* *}=p<0.01$ (Mann Whitney test). Data based on Van der Fits et al. (1999b). 
perturbation. For instance, the en bloc pattern is more frequently selected during vigorous and sudden perturbation of balance by a moving seat surface than during the small perturbation associated with voluntary reaching movements (Fig. 2). The data suggest that the age of 6 months is another period of transition in the development of postural control during which the second level of control becomes functionally active.

The idea that the age of 6 months can be regarded an age of transition is supported by findings on postural adjustments during reaching. Van der Fits et al. (1999b), who longitudinally studied postural development during infancy, reported that postural activity during reaching is characterized by a transient phase at 6 months, during which only a few muscles participate in postural control. Fallang et al. (2000), who assessed postural behavior during reaching in supine by means of kinematics of the reaching arm and kinetics of the center of pressure (COP), found that the coupling between reaching and the COP present at 4 months had almost disappeared at 6-months. The data indicate that during the transition at 6 months - in terms of the neuronal group selection theory (Hadders-Algra, 2000)-children shift from the phase of primary variability, in which motor possibilities are actively explored without precise adaptation to environmental constraints, to the phase of secondary variability in which children gradually learn to adapt motor activity to the specifics of the situation. Interestingly, 6 months is the age when significant functional activity of the frontal cortices emerges

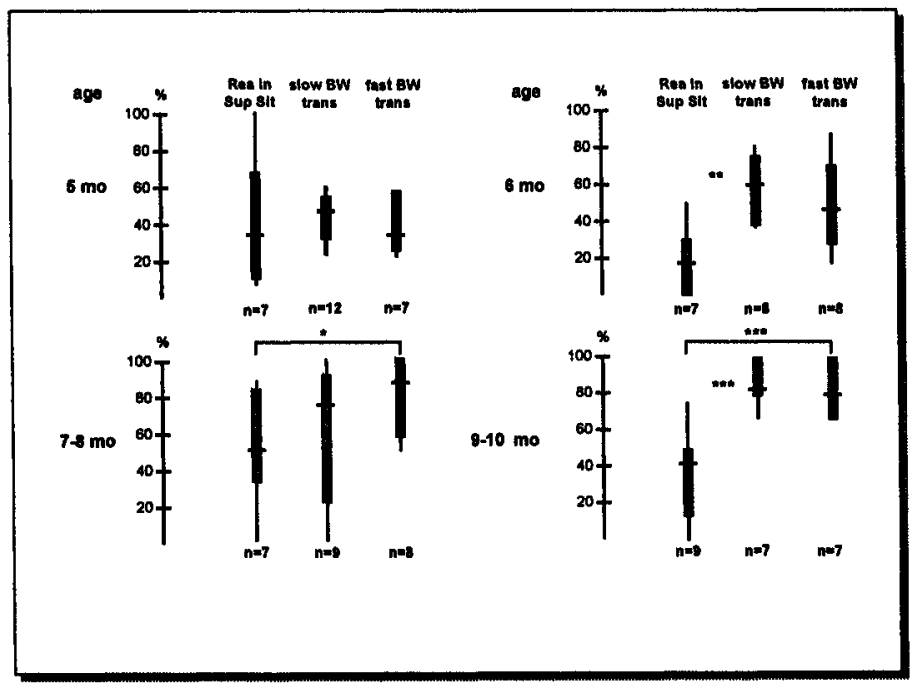

Fig. 2: Rates of occurrence of the direction specific ' $N E+L E$ ' pattern in various conditions at various ages in typically developing infants. Note that the data are derived from two different studies. The data on postural activity during reaching in supported sitting (Rea in Sup Sit) are from the Van der Fits et al. study (1999b); the data on postural adjustments during backward translations (BW trans) are from the study of Hadders-Algra et al. study (1996a). The data are presented by ranges (vertical bars), interquartile ranges (boxes), and median values (horizontal bars). ${ }^{*}=p<0.05,{ }^{* *}=p<0.01$ (Mann Whitney test). Mo $=$ months 
(Chugani, 1998). Six months, i.e. the age of transition, is also the age when infants generally learn to sit independently (Piper \& Darrah, 1994), meaning that the development of independent sitting is not dependent on the ability to adapt postural activity to the specifics of the situation accurately. The only requirement for the development of independent sitting is-from a postural control point of view-the ability to generate directionspecific postural adjustments (see Brogren Carlberg \& Hadders-Algra and Van der Heide \& HaddersAlgra, this issue).

\section{Temporal organization of postural adjustments during early infancy}

During early infancy, postural adjustments are characterized not only by variation in the muscles that participate in the adjustment but also by variation in the absolute and relative timing of muscle recruitment (Hadders-Algra et al., 1996a; Van der Fits et al., 1999b; Hedberg et al., 2005). Within the variation in timing, however, two developmental trends can be distinguished. First, during the first half year of postnatal life, a mild dominance of top-down recruitment of directionspecific muscles exists, especially during reaching movements (Van der Fits et al., 1999a). Second, latencies to the onset of direction-specific dorsal trunk and leg muscles (during forward body sway) are much shorter than latencies to the onset of the ventral muscles (during backward body sway). The direction-specific dorsal muscles are also recruited more often than direction-specific ventral muscles (Hadders-Algra et al., 1996a; Hedberg et al., 2005).

Two explanations can be offered for the developmental differences in dorsal- and ventral-muscle activity. First, the infant is forward oriented during daily life. Therefore, the infant will experience more postural behavior requiring dorsal muscle activity than [it will] postural behavior requesting the recruitment of the ventral muscles. This experiential difference might be one of the factors explaining why dorsal postural muscle activity 'matures' faster than ventral postural activity does.

Second, the neural circuitries controlling the activity of the dorsal postural muscles differ from those controlling the ventral postural muscles (Dietz, 1992; Hadders-Algra et al., 1998). The two postural systems differ in particular in the degree to which they are affected by supraspinal activity. Supraspinal modulation of the dorsal postural muscles in trunk and leg is less than that of the corresponding ventral muscles (Brogren et al., 2001).

Another interesting aspect of early postural behavior is that it is characterized by a virtual absence of antagonistic co-activation (HaddersAlgra et al., 1996a; Van der Fits et al., 1999b; Hedberg et al., 2005). This lack means that the development of postural adjustments differs in this respect from the development of other motor functions, such as reaching and walking, for which early phases are characterized by a high degree of antagonistic co-activation (Forssberg, 1985; Thelen \& Spencer, 1998).

\section{FROM SIX MONTHS ONWARD: SECONDARY VARIABILITY - LEARNING TO ADAPT POSTURAL ACTIVITY}

\section{Learning to fine-tune postural activity}

Between 6 and 9-10 months, sitting infants select increasingly more often the en bloc postural pattern from their repertoire. The en bloc adjustment is especially used when the risk of losing balance is high, which explains why this pattern remains the dominant pattern (a) during reaching while sitting until 18 months (Fig. 1, Van der Heide et al., 2003), (b) during external perturbations in sitting until the age of 30 months to 3 years (Hadders-Algra et al., 1998), and (c) during walking until 7 years of age (Assaiante, 1998).

A similar developmental pattern can be 
distinguished in the presence of antagonistic coactivation. Antagonistic co-activation in sitting tasks emerges around 9 months and can be observed in the neck muscles during reaching until the age of 18 months (Van der Fits et al., 1999b; Van der Heide et al., 2003) and during perturbations, inducing a backward body sway until 2 years of age (HaddersAlgra et al., 1998). Antagonistic co-activation during standing is present from the emergence of independent stance until at least 5 years of age (Forssberg \& Nashner, 1982).

From 9-10 months onward, infants start to develop the capacity to adapt postural adjustments in a subtle way, i.e., by means of adaptation of the degree of muscle contraction of the directionspecific muscles. During external perturbations eliciting a backward body sway in sitting position, infants develop (a) the ability to adapt the degree of contraction of all direction-specific muscles to the velocity of the moving seat surface, and (b) the ability to modulate the degree of contraction of the abdominal and leg muscles to the initial pelvis position (Hadders-Algra et al., 1996a). During reaching as well, the ability to adapt postural activity to body configuration prior to reaching emerges: a head that is held more upright, a more extended trunk, and a more reclined pelvis are associated with a more frequent selection of the en bloc pattern. Additionally, faster reaching movements are associated with a more frequent occurrence of the en bloc pattern (Van der Fits et al., 1999b).

The emergence of the ability to fine tune the degree of postural-muscle contraction to the specifics of the situation, the emergence of antagonistic coactivation, and that of the dominant presence of the en bloc pattern around the age of 9-10 months suggest that this age might be regarded as the third period of transition in postural development. The transition might be linked to the increase in functional activity in the parietal and frontal cortices occurring around this age (Rubinstein et al., 1989; Chugani, 1998)-a change in brain function also reflected in important changes in social-cognitive abilities (Carpenter et al., 1998). Conceivably, the postural transition around 9-10 months serves as a preparation for the development of standing and walking.

\section{Emergence of anticipatory postural activity}

Infants develop the ability to stand without support generally during the age period of 9 to 12 months (Piper \& Darrah, 1994). Yet, even when infants are able to stand only with support, their postural adjustments during stance are already characterized by the presence of a repertoire of direction-specific adjustments (Sveistrup \& Woollacott, 1996). With increasing age, increasing experience, and increasing capacity in standing behavior, infants learn to select from their repertoire of postural patterns the pattern in which most direction-specific muscles are activated (Sveistrup \& Woollacott, 1997). This observation means that the basic developmental principles for postural adjustments during standing are similar to those for adjustments during sitting. Nevertheless, the development of temporal organization of the adjustments differs for adjustments during sitting and standing. Postural adjustments during sitting at early age are characterized by a mild dominance of top-down recruitment, whereas postural adjustments during the first developmental phases of standing are characterized by a strong dominance of bottomup recruitment (Sveistrup \& Woollacott, 1996).

A study of Roncesvalles and colleagues (2004) underlines the two notions that (a) the basic functional organization of postural adjustments in stance is present from early standing behavior onward, and (b) the focus of postural control during the first phases of standing development is not cranially but caudally located. Roncesvalles and coworkers studied the development of two major balancing strategies during stance: (1) the ankle-strategy (which means that the entire body rotates about a single pivot point, the ankle-a 
strategy used for small perturbations of balance) and (2) the hip-strategy (consisting of a rapid flexion of the trunk on the thigh-a strategy used for larger balance perturbations). The youngest infants studied, infants with less than 6 months of walking experience, were able to apply both strategies. The organization of the ankle strategy changed minimally with increasing age, whereas the organization of the hip strategy changed from a rather passive method to one in which postural muscles are actively used.

Clark and colleagues (Barela et al., 1999; Metcalfte \& Clarke, 2000) investigated the use of somatosensory information during the acquisition of independent upright stance. The investigators studied infants aged 10 months to 2 years, who varied in developmental level from just being able to pull to standing until having some experience in independent walking. The infants either stood on a pedestal while touching a contact surface with one hand or stood 'hands-free'. At early standing age, when infants had no or little walking experience, the contact surface was used for mechanical support. After some weeks of walking experience, however, the infants used the contact surface as a source of sensory information, which was used in the prospective control of posture and as a means for exploring postural coordination.

The data of Barela et al. (1999) indicate that anticipatory postural control emerges at the age when children have some 6 weeks of walking experience, namely, at the age of 13-14 months. This report is in line with the findings of Witherington et al. (2002), who explicitly studied the emergence of anticipatory postural adjustments during stance and with those of van der Fits et al. (1999b), who studied anticipatory postural control in a sitting position. The data of Van der Fits et al. showed that the development of anticipatory postural control is related to the development of independent walking. The data suggest that the age of 13-14 months is another period of transition in the development of postural control, a transition during which feed-forward neural planning processes become integrated into postural control.

\section{CONCLUDING REMARKS}

Postural development starts with a repertoire of direction-specific adjustments. During the first half year of postnatal life, the repertoire is used in a largely variable way. This period is the phase of primary variability during which postural activity can be adapted to environmental constraints to a minimal extent only. Within the phase of primary variability, a period of transition can be observed around 3 months of age. During this period of transition, few postural muscles are recruited during postural activity. From 6 months onward, the phase of secondary variability starts, during which infants develop the ability to adapt postural activity to the specifics of the situation. Initially, adaptation can be accomplished only in a rather simple way by means of the selection of the en bloc pattern, but from 9-10 months onward, can also be performed in a more subtle way by means of an adaptation of the degree of muscle contraction of the directionspecific muscles. Around 13-14 months, anticipatory postural adjustments emerge, suggesting that at this age, infants develop the ability to integrate feedforward control into postural management.

It can be concluded that the development of postural adjustments during infancy is characterized by four periods of transition occurring at the ages of 3, 6, 9-10, and 13-14 months. Of these transitions, the one at 6 months-during which infants move from the phase of non-adaptive primary variability to the phase of adaptive secondary variability - can be regarded the major one.

\section{ACKNOWLEDGMENTS}

I thank Eva Brogren Carlberg and Victorine de Graaf-Peters for their critical and valuable remarks 
on a previous draft of this manuscript. Lidy KingmaBalkema is gratefully acknowledged for technical assistance in the preparation of the figures.

\section{REFERENCES}

Assaiante C. 1998. Development of locomotor balance control in healthy children. Neurosci Biobehav Rev 22: 527-532.

Barela JA, Jeka JJ, Clark JE. 1999. The use of somatosensory information during the acquisition of independent upright stance. Infant Behav Dev 22: 87-102.

Bernstein N. 1935. The problem of the interrelation of coordination and localization. Republished in: Whiting HTA, ed, Human Motor Actions. Bernstein Reassessed. Amsterdam, the Netherlands: Elsevier Science Publishers B.V., 1984: 77-119.

Brogren EC, Hadders-Algra M. 2005. Postural dysfunction in children with cerebral palsy: some implications for management. Neural Plast 12: 149-158, this issue.

Brogren E, Forssberg H, Hadders-Algra M. 2001. The influence of two different sitting positions on postural adjustments in children with spastic diplegia. Dev Med Child Neurol 43: 534-546.

Carpenter M, Nagell K, Tomasello M. 1998. Social cognition, joint attention, and communicative competence from 9 to 15 months of age. Monogr Soc Res Child Dev 63: 1-143.

Chugani HT. 1998. A critical period of brain development: studies of cerebral glucose utilization with PET. Prev Med 27: 184-188.

Dietz V. 1992. Human neuronal control of automatic functional movements. Interaction between central programs and afferent input. Physiol Rev 72: 33-69.

Edelman GM. 1989. Neural Darwinism. The theory of neuronal group selection. Oxford, UK: Oxford University Press; 371 .

Fallang B, Hadders-Algra M. 2005. Postural behavior in children born preterm. Neural Plast 12: 103110 , this issue.

Fallang B, Saugstad OD, Hadders-Algra M. 2000. Goal directed reaching and postural control in supine position in healthy infants. Behav Brain Res 115: 9-18.

Forssberg H. 1985. Ontogeny of human locomotor control. I. Infant stepping, supported locomotion and transition to independent locomotion. Exp
Brain Res 57: 480-493.

Forssberg H, Nashner LM. 1982. Ontogenetic development of postural control in man: adaptation to altered support and visual conditions during stance. J Neurosci 2: 545-552.

Geuze RH. 2005. Postural control in children with Developmental Coordination Disorder. Neural Plast 12: 111-124, this issue.

Gramsbergen A. 2005. Postural control in man: the phylogenitic perspective. Neural Plast 12: 5-16, this issue.

Grillner S, Deliagina T, Ekeberg Ö, EI Manira A, Hill RH, Lansner A, et al. 1995. Neural networks that co-ordinate locomotion and body orientation in lamprey. Trends Neurosci 18: 270-279.

Hadders-Algra M. 2000. The Neuronal Group Selection Theory: an attractive framework to explain variation in normal motor development. Dev Med Child Neurol 42: 566-572.

Hadders-Algra M. 2004. General movements: a window for early identification of children at high risk of developmental disorders. J Pediatr 145: S12-S18.

Hadders-Algra M, Brogren E, Forssberg H. 1996a. Ontogeny of postural adjustments during sitting in infancy: variation, selection and modulation. $\mathbf{J}$ Physiol 493: 273-288.

Hadders-Algra M, Brogren E, Forssberg H. 1996b. Training affects the development of postural adjustments in sitting infants. J Physiol 493: 289298.

Hadders-Algra M, Brogren E, Forssberg H. 1998. Development of postural control-differences between ventral and dorsal muscles? Neurosci Biobehav Rev 22: 501-506.

Hadders-Algra M, Van Eykern LA, Klip-Van den Nieuwendijk, Prechtl HFR. 1992. Developmental course of general movements in early infancy. II EMG correlates. Early Hum Dev 28: 231-251.

Hakamada S, Hayakawa F, Kuno K, Tanaka R. 1988. Development of the monosynaptic reflex pathway in the human spinal cord. Brain Res 42: 239-246.

Harbourne RT, Guiliani C, MacNeela J. 1993. A kinematic and electromyographical analysis of the development of sitting posture in infants. Dev Psychobiol 26: 51-64.

Hedberg $\AA$, Brogren Carlberg E, Forssberg $H$, Hadders-Algra M. 2005. Development of postural adjustments in sitting position during the first half year of life. Dev Med Child Neurol 47:312-320.

Hedberg A, Forssberg H, Hadders-Algra M. 2004. 
Postural adjustments due to external perturbations during sitting in 1-month-old infants: evidence for the innate origin of direction-specificity. Exp Brain Res 157: 10-17.

Hirschfeld H, Forssberg H. 1992. Phase-dependent modulations of anticipatory postural activity during human locomotion. J Neurophysiol 66: 12-19.

Hirschfeld H, Forssberg H. 1994. Epigenetic development of postural responses for sitting during infancy. Exp Brain Res 97: 528-540.

Horak FB, Nashner LM. 1986. Central programming of postural movements: adaptation to altered support-surface configurations. J Neurophysiol 55: $1369-1381$.

Massion J. 1992. Movement, posture and equilibrium -interaction and coordination. Progr Neurobiol 38: 35-56.

Massion J, Alexandrov A, Frolov A. 2004. Why and how are posture and movement coordinated? Progr Brain Res 143: 13-27.

Massion J, Ioffe M, Schmitz C, Viallet F, Gantcheva R. 1999. Acquisition of anticipatory postural adjustments in a bimanual load-lifting task: normal and pathological aspects. Exp Brain Res 128: 229-235.

Macpherson JM. 1994. Changes in postural strategy with inter-paw distance. J Neurophysiol 71: 931941.

Metcalfe JS, Clark JE. 2000. Sensory information affords exploration of posture in newly walking infants and toddlers. Infant Behav Dev 23: 391405.

Piper MC, Darrah J. 1994. Motor assessment of the developing infant. Philadelphia, Pennsylvania, USA: W.B. Saunders Company; 210.

Prechtl HFR. 1984. Continuity and change in early neural development. In: Prechtl HFR, ed, Continuity of Neural Functions from Prenatal to Postnatal Life. Clinics in Developmental Medicine 94; Oxford, Blackwell Scientific Publishers Ltd; 1-15.

Roncesvalles MN, Woollacott MH, Brown N, Jensen JL. 2004. An emerging postural response: is control of the hip possible in the newly walking child? J Mot Behav 36: 147-159.

Rubinstein M, Denays R, Ham HR, Piepsz A, VanPachterbeke T, Haumont D, et al. 1989. Functional imaging of brain maturation in humans using lodine-123 Idoamphetamine and SPECT. J
Nucl Med 30: 1982-1985.

Schmitz C, Martin N, Assaiante C. 2004. Building anticipatory adjustment during childhood: a kinematic and electromyographic analysis of unloading in children from 4 to 8 years of age. Exp Brain Res 142: 354-364.

Sveistrup H, Woollacott MH. 1996. Longitudinal development of the automatic postural response in infants. J Mot Behav 28: 58-70.

Sveistrup H, Woollacott MH. 1997. Practice modifies the developing automatic postural response. Exp Brain Res 114: 33-43.

Thelen E, Spencer JP. 1998. Postural control during reaching in young infants: a dynamic systems approach. Neurosci Biobehav Rev 22: 507-514.

Touwen BCL. 1976. Neurological development in infancy. Clin Dev Med No. 59, London, UK: Heinemann Medical Books; 150.

Touwen $\mathrm{BC}$. How normal is variable, or how variable is normal? Early Hum Dev 1993. 34: 1-12.

Van der Fits IBM, Klip AWJ, Van Eykern LA, Hadders-Algra M. 1999a. Postural adjustments during spontaneous and goal-directed arm movements in the first half year of life. Behav Brain Res 106: 75-90.

Van der Fits IBM, Otten E, Klip AWJ, Van Eykern LA, Hadders-Algra M. 1999b. The development of postural adjustments during reaching in 6 to 18 months old infants: evidence for two transitions. Exp Brain Res 126: 517-528.

Van der Heide JC, Otten B, Van Eykern LA, Hadders-Algra M. 2003. Development of postural adjustments during reaching in sitting children. Exp Brain Res 151: 32-45.

Van der Heide JC, Hadders-Algra M. 2005. Postural muscle dyscoordination in children with cerebral palsy. Neural Plast 12: 125-132, this issue.

Witherington $\mathrm{DC}$, Von Hofsten $\mathrm{C}$, Rosander $\mathrm{K}$, Robinette A, Woollacott $\mathrm{MH}$, Bertenthal BI. 2002. The development of anticipatory postural adjustments in infancy. Infancy 3: 495-517.

Woollacott M. 2005. Postural dysfunction during standing and walking in children with cerebral palsy. Neural Plast 12: 139-148, this issue.

Woollacott M, Deba B, Mowatt M. 1987. Neuromuscular control of posture in the infant and child: is vision dominant. J Motor Behav 9: 167-186. 

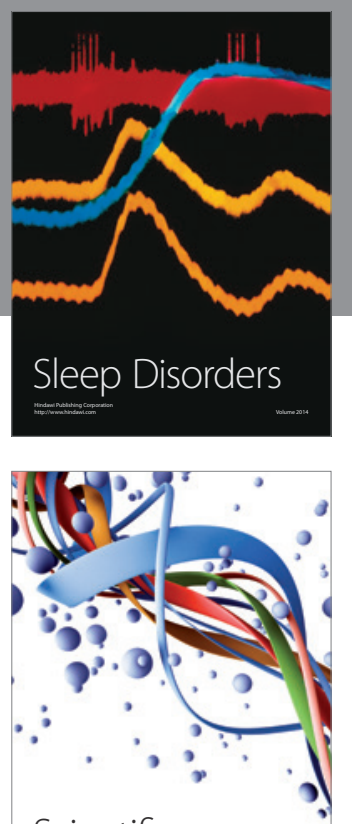

Scientifica
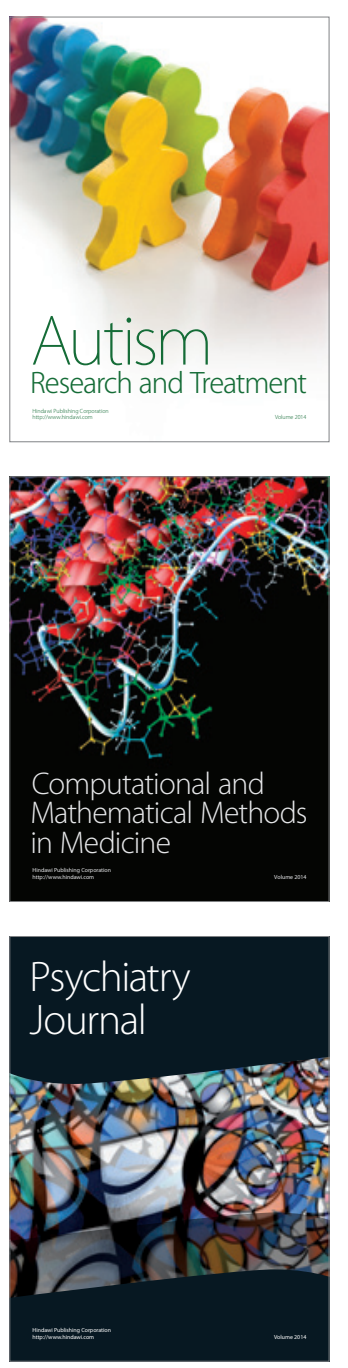
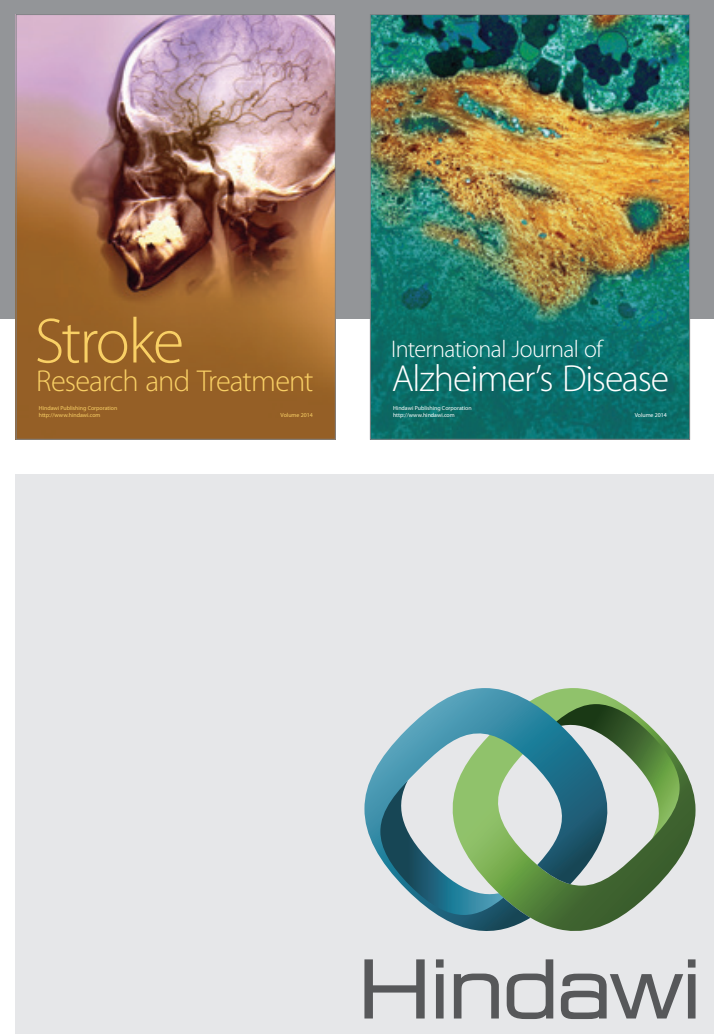

Submit your manuscripts at

http://www.hindawi.com
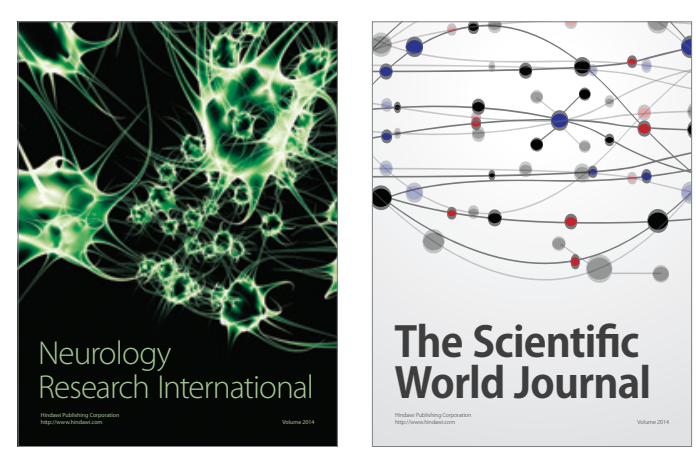

The Scientific World Journal

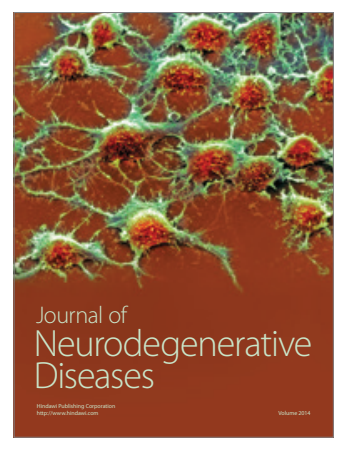

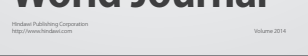

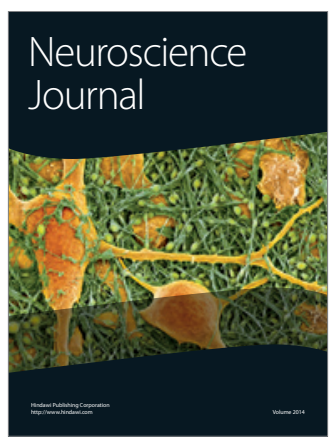

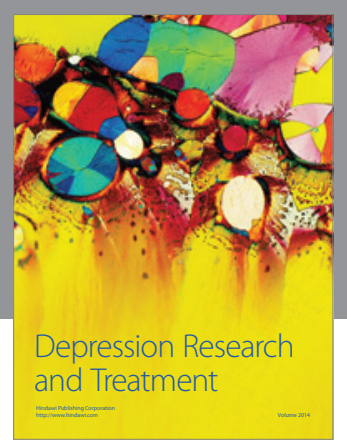
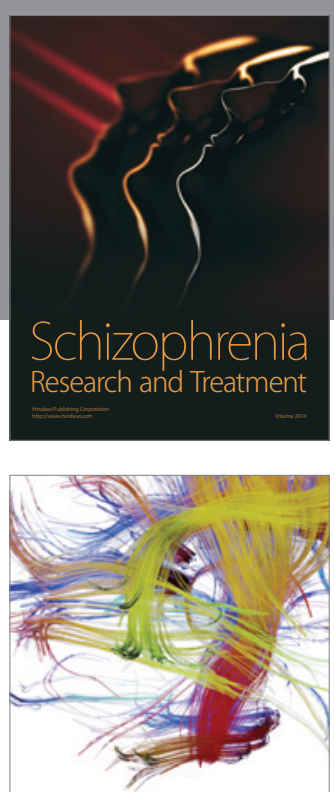

Brain Science

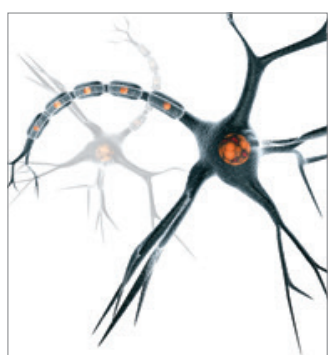

Neural Plasticity
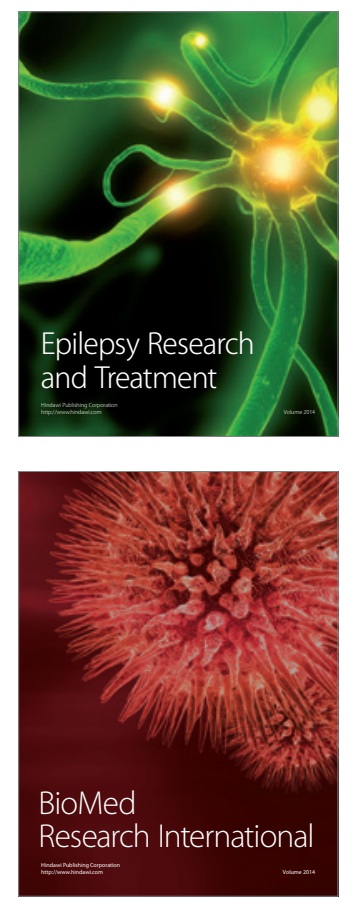

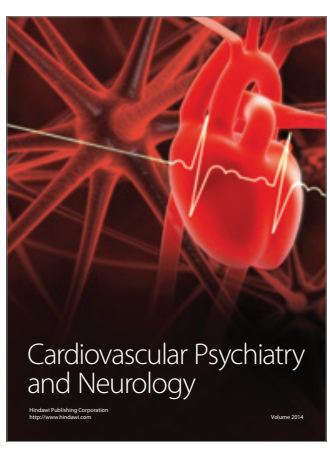

Parkinson's

Disease
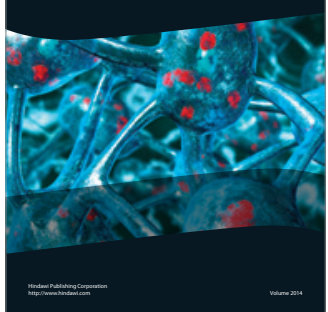